From the Princess Margaret Hospital/ Ontario Cancer Institute, Toronto;

Sunnybrook Health Sciences Centre, Toronto, Ontario; Manitoba Institute of Cell Biology, Winnipeg, Manitoba, Canada; and the Mayo Clinic, Scottsdale, AZ

Submitted April 12, 2010; accepted October 21, 2010; published online ahead of print at www.jco.org on December 28, 2010.

Supported by Celgene Corporation and the Ontario Institute for Cancer

Research.

Presented in part at the 49th Annual Meeting of the American Society of Hematology, December 7-11, 2007, Atlanta, GA; at the 50th Annual Meeting of the American Society of Hematology, December 6-9, 2008, San

Francisco, CA; and at the International Workshop for CLL October 16-18, 2009, Barcelona, Spain.

Authors' disclosures of potential conflicts of interest and author contributions are found at the end of this article.

Clinical Trials repository link available on JCO.org.

Corresponding author: Christine I. Chen, MD, MEd, FRCPC, Princess Margaret Hospital, 610 University Ave, Suite 5-220, Toronto, Ontario, Canada, M5G 2M9; e-mail:

Christine.chen@uhn.on.ca.

(C) 2010 by American Society of Clinical Oncology

0732-183X/11/2909-1175/\$20.00 DOI: $10.1200 / J C O .2010 .29 .8133$

\title{
Single-Agent Lenalidomide in the Treatment of Previously Untreated Chronic Lymphocytic Leukemia
}

Christine I. Chen, P. Leif Bergsagel, Harminder Paul, Wei Xu, Anthea Lau, Nimisha Dave, Vishal Kukreti, Ellen Wei, Chungyee Leung-Hagesteijn, Zhi Hua Li, Joseph Brandwein, Mariela Pantoja, James Johnston, Spencer Gibson, Tiffany Hernandez, David Spaner, and Suzanne Trudel

$$
\begin{array}{llllllll}
\text { A } & \text { B } & \text { S } & \text { T } & \text { R } & \text { A } & \text { C } & \text { T }
\end{array}
$$

\section{Purpose}

Lenalidomide is an oral immunomodulatory drug with multiple effects on the immune system and tumor cell microenvironment leading to inhibition of malignant cell growth. Based on encouraging reports of lenalidomide in relapsed and refractory chronic lymphocytic leukemia (CLL), we investigated the first-line use of single-agent lenalidomide in CLL.

\section{Patients and Methods}

Using a starting dose of lenalidomide $10 \mathrm{mg} / \mathrm{d}$ for 21 days of a 28-day cycle and weekly 5-mg dose escalations to a target of $25 \mathrm{mg}$, we encountered severe toxicities (tumor lysis, fatal sepsis) in the first two patients enrolled. The study was halted and the protocol amended to a more conservative regimen: starting dose of lenalidomide $2.5 \mathrm{mg}$ with monthly escalations to a target dose of $10 \mathrm{mg}$, and extended tumor lysis prophylaxis and monitoring. Gene expression profiles from patient samples before and after 7 days of lenalidomide were performed.

\section{Results}

Twenty-five patients were enrolled on the amended protocol. No further tumor lysis events were reported. Tumor flare was common (88\%) but mild. Grade 3 to 4 neutropenia occurred in $72 \%$ of patients, with only five episodes of febrile neutropenia. The overall response rate was $56 \%$ (no complete responses). Although rapid peripheral lymphocyte reductions were observed, rebound lymphocytoses during the week off-therapy were common. Lenalidomide-induced molecular changes enriched for cytoskeletal and immune-related genes were identified.

\section{Conclusion}

Lenalidomide is clinically active as first-line CLL therapy and is well-tolerated if a conservative approach with slow dose escalation is used. A lenalidomide-induced molecular signature provides insights into its immunomodulatory mechanisms of action in CLL.

\section{J Clin Oncol 29:1175-1181. (C) 2010 by American Society of Clinical Oncology}

\section{INTRODUCTION}

First-line therapies for chronic lymphocytic leukemia (CLL) range from single-agent alkylators to aggressive combination chemoimmunotherapy. Chemoimmunotherapy regimens such as fludarabine, cyclophosphamide, and rituximab (FCR) are highly active with response rates higher than 95\%. ${ }^{1}$ Based on results from the CLL8 trial, FCR is considered standard first-line therapy for selected, fit patients with CLL. ${ }^{1}$ However, FCR and other combinations have marked toxicities, are resource intensive, and remain noncurative. Hence, new agents are needed.

Lenalidomide (Revlimid; Celgene Corporation, Summit, NJ) is an oral immunomodulatory agent approved for use in multiple myeloma and myelodysplastic syndromes. Lenalidomide can directly and indirectly inhibit malignant cell growth through antiangiogenesis, direct apoptosis, and effects on the immune system and tumor microenvironment. In CLL, lenalidomide downregulates prosurvival cytokines, such as interleukin-6 (IL-6) and tumor necrosis factor-alpha (TNF- $\alpha$ ), stimulates natural killer (NK) and T-cell proliferation leading to elevated inhibitory cytokines, such as IL-2 and interferon-gamma (IFN- $\gamma$ ), upregulates B-cell activation markers, such as CD40 and CD86, inhibits stromal cell protection of leukemia cell survival, and modifies the Akt phosphorylation signaling pathway, which plays a key survival role in cancer. ${ }^{2-7}$ In addition, lenalidomide reverses CLL-induced defects in immunologic synapses, the contact points between T cells and CLL B cells that initiate the immune 
effector response. ${ }^{8}$ Hence in CLL, lenalidomide may act primarily by restoration of impaired immunosurveillance mechanisms.

Two studies using lenalidomide in CLL, both in relapsed/refractory patients, have been published. ${ }^{9,10}$ Chanan-Khan et al $^{9}$ evaluated lenalidomide at a dose and schedule used in myeloma (25 mg daily, days 1 through 21 of a 28-day schedule), attaining a response rate of $58 \%$. Tumor lysis syndrome (TLS) and tumor flare (TF), not previously noted with lenalidomide and not expected with conventional chemotherapy in CLL was reported. The MD Anderson group, using lenalidomide $10 \mathrm{mg}$ continuously dosed, reported 32\% responses and reduced toxicities (no TLS). ${ }^{10}$ Based on this evidence of clinical activity, we initiated a phase II study of first-line lenalidomide therapy in CLL. Given the reported toxicities, our study utilized a conservative dosing regimen of lenalidomide and TLS prophylaxis.

\section{PATIENTS AND METHODS}

\section{Eligibility}

Previously untreated B-cell CLL patients $\geq$ age 18 years were eligible with one or more of the following: symptomatic lymphadenopathy or organomegaly, hemoglobin lower than $110 \mathrm{~g} / \mathrm{L}$, platelets lower than $100 \times$ $10^{9} / \mathrm{L}$, lymphocyte doubling time shorter than 12 months, or significant constitutional symptoms. Required baseline values included: neutrophils higher than $1.0 \times 10^{9} / \mathrm{L}$, platelets higher than $50 \times 10^{9} / \mathrm{L}$, creatinine or bilirubin shorter than 1.5 times upper limit of normal, and aspartate or ALT lower than 2.5 times upper limit of normal. Patients gave informed consent according to institutional and university human experimentation committee requirements.

\section{Study Design and Treatment}

The original study protocol initiated lenalidomide at $10 \mathrm{mg}$ daily for 21 days of a 28-day cycle, escalating weekly by $5 \mathrm{mg}$ to a target dose of $25 \mathrm{mg}$ daily. Allopurinol $300 \mathrm{mg}$ daily for TLS prophylaxis and weekly laboratory testing was mandated for 3 weeks. Subsequent to excessive toxicities in the first two enrolled patients (see below), the protocol was amended as follows: lenalidomide starting dose reduced to $2.5 \mathrm{mg}$ daily; dose escalation modified to $2.5 \mathrm{mg}$ daily for cycle one, $5 \mathrm{mg}$ for cycle two, $10 \mathrm{mg}$ for cycle three and beyond; target dose reduced to $10 \mathrm{mg}$ daily; prolonged allopurinol prophylaxis to three cycles; extension of TLS laboratory monitoring to weekly for 8 weeks. Patients were treated until progressive disease (PD) or two cycles after complete response (CR). Dose escalation beyond $10 \mathrm{mg}$ to a maximum of $25 \mathrm{mg}$ daily was permitted for nonresponders. Dose reductions to 7.5-, 5-, and 2.5-mg daily doses were used. Treatment was delayed for grade 3 to 4 neutropenia or thrombocytopenia and resumed at lowered doses when neutrophils were $\geq$ $1.0 \times 10^{9} / \mathrm{L}$ and platelets $\geq 25 \times 10^{9} / \mathrm{L}$. Doses were held for the remainder of the cycle if midcycle neutrophils were lower than $0.5 \times 10^{9} / \mathrm{L}$ or platelets were lower than $25 \times 10^{9} / \mathrm{L}$. For grade 3 to 4 nonhematologic toxicities, treatment was withheld until $\leq$ grade 2 , then reduced doses of lenalidomide resumed. Grade 3 or worse cardiac arrythmias or any grade of desquamating rash required drug discontinuation. TF did not require dose interruption. Steroid use was permitted at the treating physician's discretion. Thrombosis prophylaxis with acetylsalicyclic acid $81 \mathrm{mg}$ daily was mandated. Use of granulocyte colony-stimulating factor (GCSF) and erythropoietin stimulating agents were allowed.

\section{Assessment of Response and Toxicity}

The primary end point was objective response to lenalidomide (CR + partial response $[\mathrm{PR}]$ ), evaluated as per the revised 1996 National Cancer Institute Working Group guidelines. ${ }^{11}$ Secondary end points were toxicity, response duration, progression-free (PFS), and overall survival (OS). Baseline evaluations included: clinical tumor measurements, CBC, liver and renal function tests, serum albumin, quantitative immunoglobulins, beta- 2 microglobulin, fluorescent in situ hybridization (FISH) analysis for 17p and 11q deletions, and trisomy 12, pregnancy testing (women of child-bearing potential), ECG, thyroid-stimulating hormone, chest x-ray, computed tomography (CT) of chest, abdomen, pelvis, and bone marrow aspirate. Immunoglobulin variable region heavy chain gene sequencing was performed with $\geq 98 \%$ homology to germ-line classified as unmutated. ${ }^{12-14}$ Elevations of $70-\mathrm{kD}$ zeta-associated protein were based on $\geq 20 \%$ threshold. Clinical and laboratory assessments (including tumor measurements by examination) were repeated on day 1 of each cycle. Laboratory monitoring for TLS was performed weekly for 8 weeks, then biweekly for 8 weeks. CT scans during therapy were optional, but mandated at baseline, at study discontinuation, and to confirm CR. Pregnancy testing was performed at baseline, weekly 4 weeks, then every 28 days.

Patients receiving one or more cycles of therapy were assessable for toxicity. Adverse events were graded using National Cancer Institute Common Toxicity Criteria version 3.0 at each visit. Patients receiving three or more cycles of therapy (time to reach target dose of $10 \mathrm{mg}$ ) were response evaluable. ${ }^{11}$ Patients were assessed 4 weeks after study discontinuation. Thereafter, follow-up was not required for patients off-study with $\mathrm{PD}$, except to document ongoing or late toxicities and death. For patients off-study with CR, PR, or stable disease (SD), follow-up continued every 3 months until progression or death.

\section{Correlative Studies}

CLL cell isolation. Blood was collected on days 1 (predosing) and 8 of cycles one and two with informed consent under a tissue banking protocol. Peripheral blood mononuclear cells (PBMCs) were prepared from whole blood by Ficoll density centrifugation. PBMCs with less than $85 \%$ purity were enriched for CLL B cells by immunoselection with anti-CD19-conjugated immunomagnetic beads (Stem Cell Technologies, Vancouver, British Columbia, Canada). Cells were aliquoted in Trizol for RNA extraction and in DMSO as viable cells for batch analysis.

\section{Microarray Experiments}

Gene expression profiling (GEP) of enriched PBMCs from day 1 (predosing) and day 8 from pooled cycles one and two for each patient was performed on 24 of 25 patient samples (one patient declined consent) using Genechip U133 Plus 2.0 (Affymetrix, Santa Clara, CA). ${ }^{15}$ Samples from cycles one and two were pooled to reduce variability and identify most consistent changes in gene expression. Three arrays were excluded from further analysis for failing more than one of five different quality control metrics defined by the BioConductor package arrayQualityMetrics (Seattle, WA; MA plots, spatial distribution of feature intensities, boxplot distribution of probe intensities, heatmap representation of the distance between arrays, relative log expression, and normalized unscaled SE plots). Expression intensities on 85 CEL files were summarized and present/absent calls made using the MAS5 algorithm (BioConductor). 28,467 probe sets called present or marginal in 20 or more of the 85 samples were used for further analysis. Each day 8 sample was normalized to the corresponding day 1 and 42 probe sets were identified as differentially expressed in the pooled day 8 samples with at least a two-fold difference in expression at $P<.05$, using Benjamini and Hochberg false discovery rate multiple testing correction.

\section{Statistical Considerations}

A two-stage design with a sample size of 25 subjects was employed, assuming a higher than $30 \%$ response rate of interest, $\alpha$ error of .05 and power of $.8 .{ }^{16}$ OS was defined from the first day of therapy to the date of death or last follow-up. PFS was defined from the first day of therapy to PD, death, or last follow-up. Response duration was measured from the time CR/PR criteria were first met until progression. Univariate analysis of response predictors was performed using Fisher's exact test and Wilcoxon rank sum test. Survival was calculated using the Kaplan-Meier method. Statistical analyses were applied using SAS 9.2 (SAS Institute, Cary, NC).

\section{RESULTS}

\section{Patients and Treatment}

Two patients were enrolled on the original protocol in October 2006. Patient 1 was a 62-year-old man, RAI stage 4 with bulky adenopathy, who initiated lenalidomide $10 \mathrm{mg}$ daily and dose-escalated to the 
target $25 \mathrm{mg}$ daily by cycle two without complication. His peripheral lymphocyte count dropped from 152 to $17.5 \times 10^{9} / \mathrm{L}$ by end of cycle one with minor reduction in adenopathy. He received TLS prophylaxis with allopurinol for 3 weeks. On day 10 of cycle 2, he developed acute renal failure consistent with TLS (elevated serum creatinine 480 umol/L, urate 1,248 umol/L, potassium $8.2 \mathrm{umol} / \mathrm{L}$, lactate dehydrogenase $398 \mathrm{U} / \mathrm{L}$ ) complicated by a myocardial infarction. Lenalidomide was discontinued and renal failure resolved with conservative management. Patient 2 was a 63 -year-old man, RAI stage 4, who initiated lenalidomide at $10 \mathrm{mg}$ daily, but did not dose-escalate further due to arthralgias, low-grade fevers, and diarrhea during week 1 . On day 11 , he developed grade 4 thrombocytopenia $\left(14 \times 10^{9} / \mathrm{L}\right)$ and grade 3 neutropenia $\left(0.8 \times 10^{9} / \mathrm{L}\right)$. Lenalidomide was withheld and GSCF instituted. He developed streptococcal sepsis with multiorgan failure and died on day 27.

In response to these toxicities, the study was halted in November 2006. Data safety monitoring board approval was obtained for protocol amendments outlined previously and enrollment reopened with 25 patients accrued from March 2007 to July 2008. Characteristics of

\begin{tabular}{|c|c|c|}
\hline \multirow[b]{2}{*}{ Characteristic } & \multicolumn{2}{|c|}{ Patients } \\
\hline & No. & $\%$ \\
\hline \multicolumn{3}{|l|}{ Sex } \\
\hline Female & 9 & 36 \\
\hline Male & 16 & 64 \\
\hline \multicolumn{3}{|l|}{ Age, years } \\
\hline Median & \multicolumn{2}{|c|}{60} \\
\hline Range & \multicolumn{2}{|c|}{$33-78$} \\
\hline 70 or older & 3 & 12 \\
\hline \multicolumn{3}{|l|}{ RAl stage } \\
\hline 0-1 & 15 & 60 \\
\hline III-IV & 10 & 40 \\
\hline Bulky adenopathy, $\geq 5 \mathrm{~cm}$ & 9 & 36 \\
\hline Enlarged spleen and/or liver & 22 & 88 \\
\hline \multicolumn{3}{|c|}{ Peripheral lymphocyte count, $\times 10^{9} / \mathrm{L}$} \\
\hline Median & \multicolumn{2}{|c|}{88.8} \\
\hline Range & \multicolumn{2}{|c|}{$2.8-220$} \\
\hline \multicolumn{3}{|l|}{ Hemoglobin level, g/L } \\
\hline Median & \multicolumn{2}{|c|}{119} \\
\hline Range & \multicolumn{2}{|c|}{$80-173$} \\
\hline \multicolumn{3}{|l|}{ Platelet level, $\times 10^{9} / \mathrm{L}$} \\
\hline Median & \multicolumn{2}{|c|}{160} \\
\hline Range & \multicolumn{2}{|c|}{$48-302$} \\
\hline \multicolumn{3}{|l|}{$\beta 2$-microglobulin, $\mathrm{nmol} / \mathrm{L}$} \\
\hline Median & \multicolumn{2}{|c|}{221} \\
\hline Range & \multicolumn{2}{|c|}{$139-626$} \\
\hline \multicolumn{3}{|l|}{$\mathrm{LDH}$} \\
\hline Median & \multirow{2}{*}{\multicolumn{2}{|c|}{$\begin{array}{c}207 \\
139-1,000\end{array}$}} \\
\hline Range & & \\
\hline Direct antiglobulin test positive & 4 & 16 \\
\hline CD38 positive & 11 & 44 \\
\hline ZAP-70 elevated & 15 & 60 \\
\hline Unmutated IgVH & 18 of $24^{*}$ & 75 \\
\hline FISH 11q or $17 p$ deletion & 8 & 32 \\
\hline $\begin{array}{l}\text { Abbreviations: LDH, lactate deh } \\
\text { region heavy chain gene; FISH, } \\
\text { associated protein. } \\
{ }^{*} \text { One sample unreadable. }\end{array}$ & $\begin{array}{l}\text { t, immuno } \\
\text { u hybridiza }\end{array}$ & \\
\hline
\end{tabular}

patients are listed in Table 1. Median age was 60 years (range, 33 to 78 years). Most patients had nodal disease (67\%), organomegaly $(88 \%)$, and elevated serum $\beta 2$-microglobulin ( $>169 \mathrm{nmol} / \mathrm{L} ; 84 \%$ ). Fourteen patients had unfavorable FISH cytogenetics: deletion 11q (five patients; $20 \%$ ), deletion $17 \mathrm{p}$ (three patients; 12\%), trisomy 12 (six patients; 24\%).

\section{Dose Modifications/Discontinuation}

A median of 18 cycles (range, 2 to 33 cycles) were administered (total 470 cycles for all patients). Median dose reached was 15 mg daily (range, 2.5 to $25 \mathrm{mg}$ ). Eight patients have discontinued therapy: seven (28\%) for drug-related toxicity, one (4\%) for nonresponse (SD after 10 cycles). Toxicity leading to discontinuation included: prolonged cytopenias $(\mathrm{n}=3)$, recurrent infections $(\mathrm{n}=$ $1)$, disseminated herpes zoster $(\mathrm{n}=1)$, grade 3 rash $(\mathrm{n}=1)$, persistent grade 2 diarrhea $(n=1)$. Dose reductions were required in eight patients (32\%), all due to cytopenias. Dose delays occurred in $11.9 \%$ of all 470 cycles, $70 \%$ due to cytopenias, $7.1 \%$ other toxicities, and the rest for clinic/patient holidays.

\section{Toxicity}

Table 2 outlines lenalidomide-related toxicities. The most common nonhematologic toxicities were $\mathrm{TF}(88 \%)$, fatigue $(72 \%)$, and rash (64\%). TF, defined by painful and/or enlarging lymph node(s), presented most commonly in the first week of cycle one. Repeat flare symptoms occurred in $26 \%$ of subsequent cycles on resuming drug after cycle day 22 through 28 off-therapy. TF was mild (grade 1 to 2 ) and frequently accompanied by allergic-type symptoms, such as nasal congestion, coryza, scalp itching. Most TF symptoms resolved spontaneously, but eight patients (32\%) required a brief course of steroids (typically prednisone 25 to $50 \mathrm{mg}$ daily for 5 to 10 days) on at least one occasion. Rashes were common (64\%) and heterogeneous in presentation (diffuse maculopapular; nodular; urticarial). One patient developed grade 3 maculopapular rash with concurrent HSV1 isolated from skin, requiring hospitalization for symptom control. Fifteen patients $(60 \%)$ developed infections, mostly upper respiratory, skin, and sinus infections. There were six grade 3 to 4 infections in four patients (excluding febrile neutropenia): pneumonia $(n=2)$, cellulitis $(n=2)$, disseminated zoster $(n=1)$, nonspecific febrile illness $(\mathrm{n}=1)$.

Grade 3 to 4 neutropenia developed in 18 patients (72\%), with five episodes of febrile neutropenia. Eight patients (32\%) required GCSF support on at least one occasion. Of 470 cycles administered to all patients, $14.7 \%$ were complicated by grade 3 to 4 neutropenia and $3 \%$ by grade 3 to 4 thrombocytopenia (Fig 1 ).

\section{Efficacy}

Of 25 subjects, 14 (56\%) achieved a PR, 10 (40\%) achieved SD, with no CR observed. Twenty (80\%) achieved $\geq 50 \%$ reduction in peripheral lymphocytes. Although lymphocyte reductions often occurred within the first week of therapy, rebound lymphocytoses were frequently noted during days 22 through 28 off therapy (Fig 2). Nine $(50 \%)$ of 18 patients with nodal disease at baseline achieved complete resolution of adenopathy on therapy. Median time to response of both peripheral lymphocytes and bidimensional disease was 4.9 months (range, 1.8 to 19.6 months). For the 14 responders, median duration of response was 16.6 months (range, 5.0 to 28.7 months). One patient who discontinued therapy due to recurrent infections showed nodal progression on end-of-study CT scans. He subsequently died 30 weeks 


\begin{tabular}{|c|c|c|c|c|c|c|}
\hline \multirow[b]{3}{*}{ Event } & \multicolumn{6}{|c|}{ Adverse Events by Grade } \\
\hline & \multicolumn{2}{|c|}{$\begin{array}{c}\text { All } \\
\text { Related }\end{array}$} & \multicolumn{2}{|c|}{3} & \multicolumn{2}{|c|}{4} \\
\hline & No. & $\%$ & No. & $\%$ & No. & $\%$ \\
\hline \multicolumn{7}{|l|}{ Nonhematologic } \\
\hline Tumor flare & 22 & 88 & & & & \\
\hline Fatigue & 18 & 72 & & & & \\
\hline Rash & 16 & 64 & 1 & 4 & & \\
\hline Myalgia & 10 & 40 & & & & \\
\hline Pruritis & 8 & 32 & & & & \\
\hline Diarrhea & 8 & 32 & & & & \\
\hline Constipation & 6 & 24 & & & & \\
\hline Sensory neuropathy & 4 & 16 & & & & \\
\hline Leg edema & 3 & 12 & & & & \\
\hline Dizziness & 3 & 12 & & & & \\
\hline Generalized muscle weakness & 3 & 12 & & & & \\
\hline Dyspnea & 3 & 12 & & & & \\
\hline \multicolumn{7}{|l|}{ Metabolic } \\
\hline Elevated creatinine & 11 & 44 & & & & \\
\hline Hypocalcemia & 11 & 44 & 1 & 4 & & \\
\hline Hyperkalemia & 10 & 40 & & & & \\
\hline Hypophosphatemia & 10 & 40 & 2 & 8 & & \\
\hline Elevated AST or ALT & 9 & 36 & & & & \\
\hline Hypernatremia & 5 & 20 & & & & \\
\hline Hyponatremia & 4 & 16 & 1 & 4 & & \\
\hline Infection & 15 & 60 & 7 & 28 & 2 & 8 \\
\hline Febrile neutropenia & 5 & 20 & 3 & 12 & 2 & 8 \\
\hline Skin & 4 & 16 & 2 & 8 & & \\
\hline Upper respiratory/sinus & 2 & 8 & & & & \\
\hline Wound & 1 & 4 & & & & \\
\hline Lung & 1 & 4 & 1 & 4 & & \\
\hline Other & 2 & 8 & 1 & 4 & & \\
\hline \multicolumn{7}{|l|}{ Hematologic } \\
\hline Neutropenia & 18 & 72 & 10 & 40 & 8 & 32 \\
\hline Anemia & 6 & 24 & 4 & 16 & 1 & 4 \\
\hline Thrombocytopenia & 9 & 36 & 3 & 12 & 4 & 16 \\
\hline
\end{tabular}

after study discontinuation with Richter's transformation. On univariate analysis, clinical features, such as bulky nodal disease $(\geq 5$ $\mathrm{cm}$ ), organomegaly, advanced RAI stage, or TF symptoms, did not predict for response. Of the laboratory features analyzed (ie, baseline hemoglobin, WBC, platelets, neutrophils, lymphocytes, albumin, lactate dehydrogenase, beta- 2 microglobulin, direct antiglobulin test, $70-\mathrm{kD}$ zeta-associated protein, CD38, FISH cytogenetics), only the presence of higher prestudy neutrophils significantly correlated with response (median $6.0 \times 10^{9} / \mathrm{L}$ in responders $v 2.7 \times 10^{9} / \mathrm{L}$ in nonresponders; $P=.01)$.

\section{Survival}

Three patients have died at a median follow-up of 20.7 months (range, 12.8 to 30.6 months). For all 25 patients, the estimated twoyear OS was $92 \%$ (95\% CI, $81 \%$ to $100 \%)$ and PFS was $89 \%$ (95\% CI, $74 \%$ to $100 \%$; Fig 3 ).

\section{Lenalidomide-Induced Gene Expression Changes}

A lenalidomide-induced signature representing 42 differentially expressed genes with at least a two-fold difference in expression was

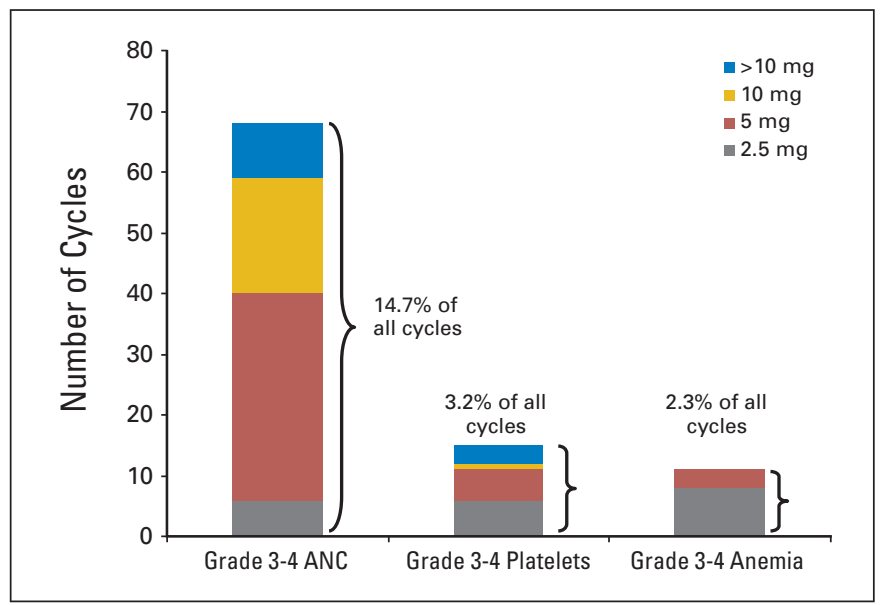

Fig 1. Proportion of cycles affected by grade 3 to 4 cytopenias. Of a total of 470 cycles administered to all patients, $14.7 \%$ were complicated by grade 3 to 4 neutropenia, $3.2 \%$ grade 3 to 4 thrombocytopenia, and $2.3 \%$ grade 3 to 4 anemia. For each grade 3 to 4 cytopenia (represented by each bar), the proportion occurring at each dose level is depicted by the different colors. The majority of grade 3 to 4 cytopenias occurred at low doses 2.5 to $5 \mathrm{mg}$ daily. ANC, absolute neutrophil count.

identified (Fig 4). Twenty-six genes were upregulated (median, 2.15fold; range, 2.0- to 3.1-fold); 16 genes downregulated (median, 2.13fold; range, 2.0- to 3.9-fold).

As expected, the lenalidomide-induced signature of gene expression change was significantly enriched for immune-associated genes, including TNF- $\alpha$, lymphocyte activation gene-3 (LAG3), and IFNinduced genes, such as 2,5-oligoadenylate synthetase. In addition to immune-related genes, the most highly upregulated gene in our analysis was tropomyosin 2, a cytoskeletal-regulatory protein. ${ }^{17,18}$ CD40 increased 1.4 fold $(P<.001)$, CD86 increased modestly (1.2-fold, $P<.05)$, with no consistent change in CD80. Immunofluorescence cell surface expression of TNF- $\alpha$, LAG3, and tropomyosin 2 , the three most highly upregulated genes identified, was performed to confirm the microarray findings (Appendix Figs A1, A2, A3, online only).

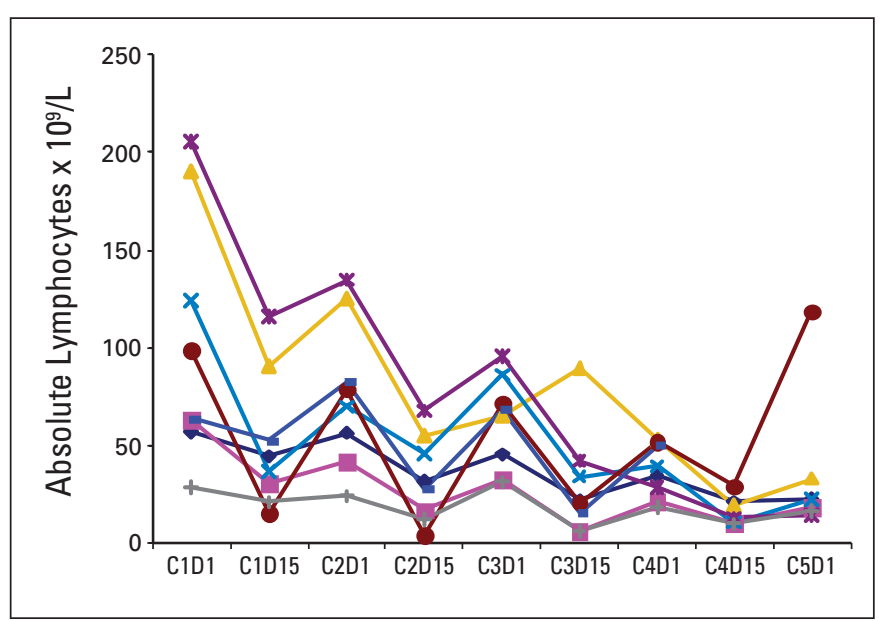

Fig 2. Cyclic rebound of peripheral lymphocytosis. Although significant reductions in absolute peripheral lymphocyte counts can be seen by day 15 of each cycle, a rebound increase in lymphocytes can be seen in some patients before start of next cycle. This figure demonstrates this rebound in eight representative patients. 


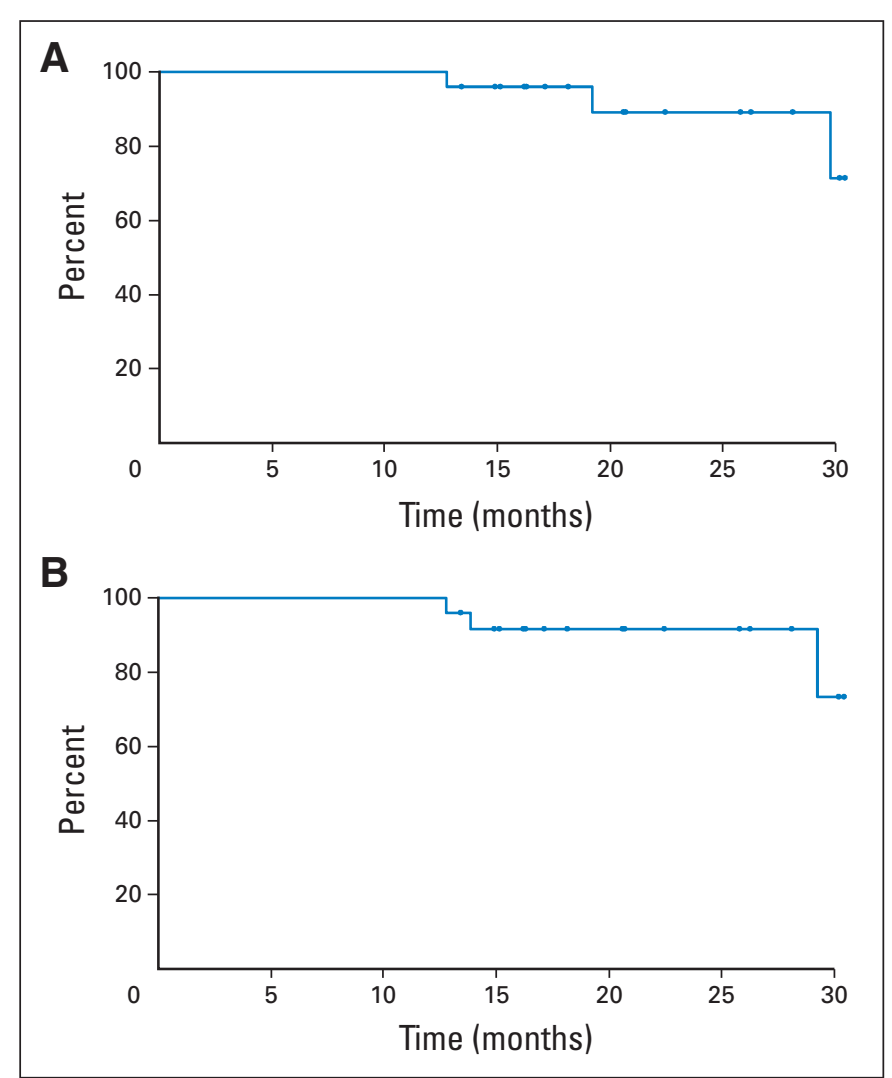

Fig 3. Kaplan-Meier survival curves. (A) Progression-free survival in months. (B) Overall survival in months.

\section{DISCUSSION}

To our knowledge, our study is the first published report of lenalidomide for treatment-naive CLL. Our response rate of 56\% is evidence of remarkable antileukemia activity, given the low doses of singleagent lenalidomide used. It is comparable to reported response rates of up to $58 \%$ using lenalidomide in relapsed/refractory CLL ${ }^{9,10}$ and with other first-line single-agent regimens evaluated in randomized trials (fludarabine $60 \%$ to $83 \%,{ }^{19-22}$ bendamustine $68 \%,{ }^{23}$ alemtuzumab $83 \%,{ }^{24}$ and chlorambucil $33 \%$ to $\left.72 \%\right) .{ }^{19,22}$ In our patients, reductions of peripheral lymphocytoses were rapid in onset, but rebound observed during the 7 days off drug at the end of each cycle, suggests that at these low doses, lenalidomide has a suppressive rather than cytotoxic effect. At a median follow-up of 20.7 months, $68 \%$ of patients remain on drug and progression free. The occurrence of responses as late as 19 months after initiation of lenalidomide suggests that prolonged therapy may be necessary to maximize responses. This prolonged, low-dose suppressive approach to CLL therapy contrasts starkly with aggressive, multidrug combinations, such as FCR, and should be evaluated further in the elderly or those with multiple comorbidities where FCR may not be appropriate.

Based on reports of TLS and TF with lenalidomide in relapsed/ refractory CLL, ${ }^{9}$ our original protocol used a starting dose of lenalidomide lower than doses used in earlier studies, including a plan to slowly escalate doses. Early, severe toxicities in the first two patients were unexpected, although reports of similar events in patients with CLL have now appeared, with growing recognition that lenalido-

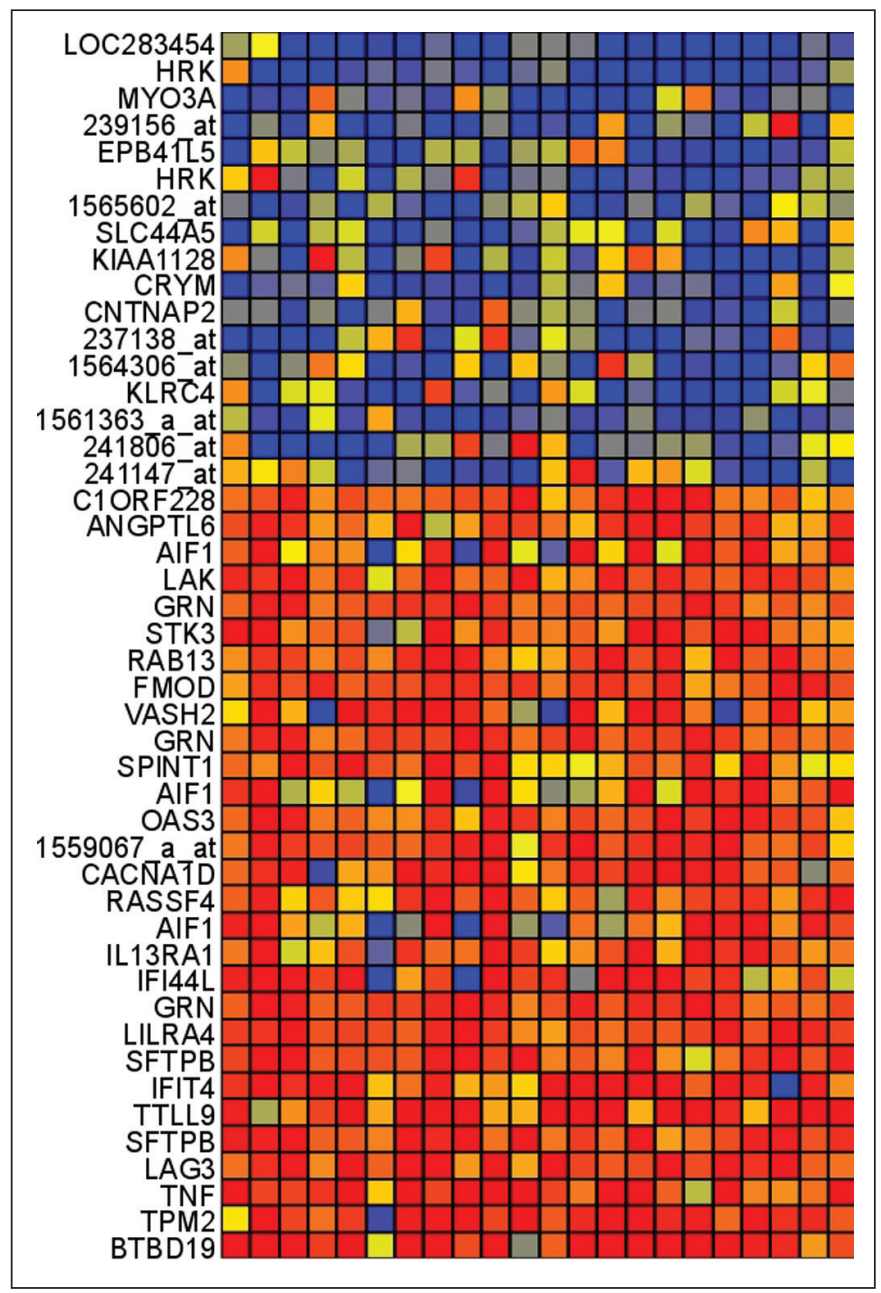

Fig 4. Lenalidomide-induced molecular changes. Molecular responses were evaluated on days 1 (before initiation of therapy) and 8 (after lenalidomide dosing) for cycles one and two. A volcano plot identified 42 probe sets with a greater than two-fold difference in expression at day 8 with a $P$ lower than .05 using Benjamini and Hochberg false detection rate multiple test correction. The heatmap shows the change in gene expression (red three-fold up, and blue three-fold down) of genes at day 8 compared to day 1 in 22 patients (pooling the data from cycle one and two for each patient). Twenty-six probe sets were upregulated and sixteen probe sets were downregulated. In general, we observed that the same direction of lenalidomide-induced changes in gene expression (down or upregulation) was found in the majority of patients.

mide's safety record in approved indications, such as myeloma, cannot be routinely generalized to CLL. ${ }^{5,25}$ After protocol amendments that reduced the initial lenalidomide dose to one tenth that used in myeloma and slowed the dose escalation schedule, no further episodes of TLS were seen. However, high rates of TF $(88 \%)$, a phenomenon not commonly reported with conventional chemotherapy in CLL, were seen. TF reactions were mild, generally self-limited, and did not lead to dose reductions or study withdrawals. In relapsed CLL, lenalidomide causes TF in $12 \%$ to $58 \%$ of patients and appears more severe with higher starting doses. ${ }^{9,10,25}$ The higher incidence of TF in our study suggests that chemotherapy-naive patients, unlike pretreated patients, have robust immune systems responsive to the immunostimulatory effects of lenalidomide.

As with lenalidomide use in myeloma, hematologic toxicities were the most common severe toxicities and the most frequent cause 
for dose reductions in our study. Dose reduction, rather than GCSF use, was the most common approach taken for neutropenia management. Considering the already low doses of lenalidomide used in our study, this approach may compromise response. Lower baseline neutrophil counts, taken as a marker of compromised normal hematopoiesis, predicted for poor response and suggests that more aggressive use of GCSF to avoid dose reductions may optimize efficacy.

Although lenalidomide has significant clinical activity in CLL, its exact molecular mechanisms remain unclear. Using GEP analysis of paired patient samples from baseline and after 7 days of lenalidomide, we identified a lenalidomide-induced signature enriched for genes involved in cytoskeletal organization and immune response. Tropomyosin 2, the most differentially upregulated gene by GEP, was also shown by immunofluorescence to increase in cell surface expression after lenalidomide exposure. Tropomyosin 2 regulates actin organization and cell function, similar to that of Rho GTPase, a recently identified molecular target of immunomodulatory drugs. ${ }^{26}$ Upregulated genes with immune-related function included $T N F-\alpha, L A G-3$, and $I F N$-induced genes. We demonstrated that lenalidomide upregulates TNF- $\alpha$ gene expression and enhances intracellular TNF- $\alpha$ expression in activated CLL cells, consistent with prior reports of elevated serum TNF- $\alpha$ /TNF receptor- 1 in patients with CLL after lenalidomide therapy. ${ }^{10,27}$ LAG-3, upregulated in both mRNA and cell surface expression in our studies, is an MHC class II binder with a complex role in controlling dendritic and T-cell functions. ${ }^{28,29}$ LAG-3 activates antigen-presenting cells leading to their maturation and migration into lymphoid organs and increases antigen-specific responses in vivo. ${ }^{30,31}$ Human recombinant LAG-3 is currently undergoing clinical testing as an adjuvant for cancer vaccines. ${ }^{32,33}$ Increased expression of IFN-induced genes in our drug-induced signature also suggests that enhancement of the IFN response may be fundamental to lenalidomide's antitumor activity. ${ }^{34}$ Although we identified CD40 upregulation, we did not observe consistent upregulation of other costimulatory molecules, such as CD80 and CD86 as previously reported, although alternative methodologies for confirmation were not performed. ${ }^{5,27}$

In conclusion, lenalidomide is an active first-line treatment option for CLL that is well-tolerated if a conservative approach with low doses and slow dose-escalation is used. Failure to achieve CR and observations of rebound lymphocytosis suggest that low-dose lenalidomide may require continuous daily dosing and combination with other synergistic agents for optimal effect. Lenalidomide-induced gene expression changes provide direction for future investigations into the mechanisms of action of this novel agent.
AUTHORS' DISCLOSURES OF POTENTIAL CONFLICTS OF INTEREST

Although all authors completed the disclosure declaration, the following author(s) indicated a financial or other interest that is relevant to the subject matter under consideration in this article. Certain relationships marked with a "U" are those for which no compensation was received; those relationships marked with a "C" were compensated. For a detailed description of the disclosure categories, or for more information about ASCO's conflict of interest policy, please refer to the Author Disclosure Declaration and the Disclosures of Potential Conflicts of Interest section in Information for Contributors.

Employment or Leadership Position: None Consultant or Advisory Role: Christine I. Chen, Celgene Corporation (C), Roche (C), Ortho Biotech (C); P. Leif Bergsagel, Celgene Corporation (C), Genentech (C), Amgen (C), Novartis (C), Centocor (C); Joseph Brandwein, Celgene Corporation (C); James Johnston, Roche (C); Suzanne Trudel, Celgene Corporation (C), Ortho Biotech (C), Genentech (C), Exelexis (C) Stock Ownership: None Honoraria: Christine I. Chen, Celgene Corporation, Roche, Ortho Biotech, Genzyme; Vishal Kukreti, Celgene Corporation, Ortho Biotech, Roche; Joseph Brandwein, Celgene Corporation; James Johnston, Roche; Suzanne Trudel, Celgene Corporation, Orthobiotech Research Funding: Christine I. Chen, Celgene Corporation; Joseph Brandwein, Celgene Corporation; Suzanne Trudel, Celgene Corporation, Exelexis Expert Testimony: None Other Remuneration: None

\section{AUTHOR CONTRIBUTIONS}

Conception and design: Christine I. Chen, Suzanne Trudel Financial support: Suzanne Trudel

Administrative support: Harminder Paul, Suzanne Trudel

Provision of study materials or patients: Christine I. Chen, P. Leif Bergsagel, Vishal Kukreti, Joseph Brandwein, David Spaner Collection and assembly of data: Christine I. Chen, P. Leif Bergsagel, Harminder Paul, Wei Xu, Anthea Lau, Nimisha Dave, Ellen Wei, Chungyee Leung-Hagesteijn, Zhi Hua Li, Mariela Pantoja, James Johnston, Tiffany Hernandez, Suzanne Trudel

Data analysis and interpretation: Christine I. Chen, P. Leif Bergsagel, Harminder Paul, Wei Xu, Ellen Wei, Chungyee Leung-Hagesteijn, Zhi Hua Li, James Johnston, Spencer Gibson, David Spaner, Suzanne Trudel Manuscript writing: Christine I. Chen, P. Leif Bergsagel, Harminder Paul, Wei Xu, Anthea Lau, Nimisha Dave, Vishal Kukreti, Ellen Wei, Chungyee Leung-Hagesteijn, Zhi Hua Li, Joseph Brandwein, Mariela Pantoja, James Johnston, Spencer Gibson, Tiffany Hernandez, David Spaner, Suzanne Trudel

Final approval of manuscript: Christine I. Chen, P. Leif Bergsagel, Harminder Paul, Wei Xu, Anthea Lau, Nimisha Dave, Vishal Kukreti, Ellen Wei, Chungyee Leung-Hagesteijn, Zhi Hua Li, Joseph Brandwein, Mariela Pantoja, James Johnston, Spencer Gibson, Tiffany Hernandez, David Spaner, Suzanne Trudel

\section{REFERENCES}

1. Hallek M, Fischer $K$, Fingerle-Rowson G, et al: Addition of rituximab to fludarabine and cyclophosphamide in patients with chronic lymphocytic leukaemia: A randomized, open-label, phase 3 trial. Lancet 376:1164-1174, 2010

2. Dredge $K$, Horsfall R, Robinson S, et al: Orally administered lenalidomide is anti-angiogenic in vivo and inhibits endothelial cell migration and Akt phosphorylation in vitro. Microvasc Res 69:56-63, 2005

3. Schafer $P$, Gandhi $A$, Loveland $M$, et al: Enhancement of cytokine production and AP-1 tran- scriptional activity in $\mathrm{T}$ cells by thalidomide-related immunomodulatory drugs. J Pharm Exp Therapeutics 305:1222-1232, 2003

4. Corral L, Haslett $P$, Muller F, et al: Differential cytokine modulation and $\mathrm{T}$ cell activation by two distinct classes of thalidomide anlaogues that are potent inhibitors of TNF-alpha. J Immunol 163:380-386, 1993

5. Andritsos L, Johnson AJ, Lozanski G, et al: C: Higher doses of lenalidomide are associated with unacceptable toxicity including life threatening tumor flare in patients with chronic lymphocytic leukemia. J Clin Oncol 26:2519-2525, 2008

6. Lapalombella R, Andritsos L, Liu Q, et al: Lenalidomide treatment promotes CD154 expres- sion on CLL cells and enhances production of antibodies by normal B Cells through a PI3kinase dependent pathway. Blood 115:2619-2629, 2010

7. James D, Betty M, Mosadeghi R, et al: Lenalidomide abrogates the protective influence of nurse-like cells on primary chronic lymphocytic leukemia cells in vitro. Blood 110:916a, 2007 (abstr 3116)

8. Ramsay A, Johnson A, Lee $A$, et al: Chronic lymphocytic leukemia $T$ cells show impaired immunological synapse formation that can be reversed with an immuomodulating drug. J Clin Invest 118: 2427-2437, 2008 
9. Chanan-Khan A, Killer K, Musial L, et al: Clinical efficacy of lenalidomide in patients with relapsed or refractory chronic lymphocytic leukemia: Results of a phase II study. J Clin Oncol 24:53435349, 2006

10. Ferrajoli A, Lee B-N, Schlette $E$, et al: Lenalidomide induces complete and partial remissions in patients with relapsed and refractory chronic lymphocytic leukemia. Blood 111:5291-5297, 2008

11. Cheson BD, Bennet JM, Grever M, et al: $\mathrm{NCl}$ Working Group Guidelines for CLL: Revised guidelines for the diagnosis and treatment. Blood 87: 4990-4997, 1996

12. Amrein L, Hernandez $T$, Ferrario $C$, et al: Dasatinib sensitizes primary chronic lymphocytic leukaemia lymphocytes to chlorambucil and fludarabine in vitro. Br J Haematol 143:698-706, 2008

13. Jelinek D, Tschumper R, Geyer $S$, et al: Analysis of clonal B-cell CD38 and immunoglobulin variable region sequence status in relation to clinical outcome for B-chronic lymphocytic leukaemia. Br J Haematol 115:854-861, 2001

14. Fais F, Ghiotto F, Hashimoto S, et al: Chronic lymphocytic leukemia $B$ cells express restricted sets of mutated and unmutated antigen recpetors. J Clin Invest 102:1515-1525, 1998

15. Chng W, Schop R, Price-Troska T, et al: Geneexpression profiling of Waldenstrom macroglobulinemia reveals a phenotype more similar to chronic lymphocytic leukemia than multiple myeloma. Blood 108:2755-2763, 2006

16. Fleming $\mathrm{T}$ : One-sample multiple testing procedure for phase II clinical trials. Biometrics 38:143151, 1982

17. O'Neill G, Stehn J, Gunning P: Tropomyosins as interpreters of the signalling environment to regulate the local cytoskeleton. Semin Cancer Biol 18:35-44, 2008
18. Densham R, O'Neill E, Munro J, et al: MST kinases monitor actin cytoskeletal integrity and signal via c-Jun $\mathrm{N}$-terminal kinase stress-activated kinase to regulate p21 Wafl/Cipl stability. Mol Cell Biol 29:6380-6390, 2009

19. Catovksy D, Richards S, Matutes E, et al: Assessment of fludarabine plus cyclophosphamide for patients with chronic lymphocytic leukaemia (the LRF CLL4 Trial): A randomised controlled trial. Lancet 370:230-239, 2007

20. Flinn I, Neuberg D, Grever M, et al: Phase III trial of fludarabine plus cyclophosphamide compared with fludarabine for patients with previously untreated chronic lymphocytic leukemia: US Intergroup trial E2997. J Clin Oncol 25:793-798, 2007

21. Eichhorst B, Busch R, Hopfinger G, et al: Fludarabine plus cyclophosphamide versus fludarabine alone in first-line therapy of younger patients with chronic lymphocytic leukemia. Blood 107:885891, 2006

22. Rai K, Peterson B, Appelbaum F, et al: Fludarabine compared with chlorambucil as primary therapy for chronic lymphocytic leukemia. N Engl J Med 343:1750-1757, 2000

23. Knauf W, Lissichkov $T$, Aldaoud L, et al: Phase III randomized study of bendamustine compared with chlorambucil in previously untreated patients with chronic lymphocytic leukemia. J Clin Oncol 27:4378-4384, 2009

24. Hillman $P$, Skotnicki $A$, Robak $T$, et al: Alemtuzumab compared with chlorambucil as first-line therapy for chronic lymphocytic leukemia. J Clin Oncol 25:5616-5623, 2007

25. Moutouh-de Parseval L, Weiss L, DeLap R, et al: Tumor lysis syndrome/tumor flare reaction in lenalidomide-treated chronic lymphocytic leukemia. J Clin Oncol 25:5047, 2007
26. Xu Y, Li J, Ferguson GD, et al: Immunomodulatory drugs reorganize cytoskeleton by modulating Rho GTPases. Blood 114:338-345, 2009

27. Aue G, Njuguna $N$, Tian $X$, et al: Lenalidomideinduced upregulation of CD80 on tumor cells correlates with T-cell activation, the rapid onset of a cytokine release syndrome and leukemic cell clearance in chronic lymphocytic leukemia. Haematologica 94:1266-1273, 2009

28. Casati C, Camisaschi C, Novellino L, et al: Human lymphocyte activation gene-3 molecules expressed by activated $T$ cells deliver costimuation signal for dendritic cell activation. J Immunol 180: 3782-3788, 2008

29. Brignone C, Grygar C, Marcu M, et al: A soluble form of lymphocyte activation gene-3 (IMP321) induces activation of a large range of human effector cytotoxic cells. J Immunol 179:4202-4211, 2007

30. Baixeras E, Hard B, Miossec C, et al: Characterization of the lymphocyte activation gene 3-encoded protein: A new ligand for human leukocyte antigen class II antigens. J Exp Med 176:327-337, 1992

31. Workman C, Vignali D: The CD4-related molecule, LAG-3 (CD223), regulates the expansion of activated T cells. Eur J Immunol 33:970-979, 2003

32. Prigent $P$, El Mir S, Dreano M, et al: Lymphocyte activation gene-3 induces tumor regression and antitumor immune responses. Eur J Immunol 29: 3867-3876, 1999

33. Brignone C, Escudier B, Grygar C, et al: A phase I pharmacokinetic and biological correlative study of IMP321, a novel MHC class II agonist, in patients with advanced renal cell carcinoma. Clin Cancer Res 15:6225-6231, 2009

34. Silverman R: Viral encounters with $2^{\prime}, 5^{\prime}-$ oligoadenylate synthetase and RNase $L$ during the interferon antiviral response. J Virology 81:1272012729, 2007 